\title{
Modeling and Simulation of Bus Priority Oriented Urban Road Network Elastic Equilibrium
}

\author{
Lihua Liu', 2, a , Yaping Zhang ${ }^{1, b}$ and Yu Wei ${ }^{1, c}$ \\ ${ }^{1}$ School of Transportation Science and Engineering, Harbin Institute of Technology, Harbin 150090, \\ China \\ ${ }^{2}$ Department of Transportation Engineering, Henan University of Urban Construction, Pingdingshan, \\ 467044, China \\ aliulihuaalicia@126.com, ’zxlt0905@163.com, cweixiaoyu8899@163.com
}

Keywords: Bus Priority. Elastic Equilibrium. ABC Classification. Bi-Level Programming. VISSIM.

\begin{abstract}
Influenced from economy, population and many other factors, rail transit and elevated road have poor applicability in small and medium sized city. Maximizing application of available road resources and bus facilities, reducing the environmental pollution is the critical issue faced by current situation of urban transportation development in China. Guided by Bus Priority Oriented, utilized comprehensive techniques, such as $\mathrm{ABC}$ classification, bi-level programming model, sensitivity analysis, this paper constructed a theoretical frame of elastic equilibrium based on system optimization. Taking time and environment as optimized objectives, this paper established the Bi-Level multi-objective programming model based on travel time, and programmed with MATLAB for the calculation. Finally, the case study is illustrated. The software VISSIM was adopted to realize the simulation of the volume before and after the implementation of elastic plan in urban areas of PingDingshan. The simulation results showed the validity and feasibility of the modeling and simulation of Bus Priority Oriented urban road network elastic equilibrium.
\end{abstract}

\section{Introduction}

The elastic traffic is a great interesting topic of research at present and also the direction of future traffic development. Based on the Bus Priority Theory, the elastic traffic has all the strategic merits of the bus priority, is the allocation of resources, an effective way to solve the traffic jam problem in cities, will contribute to the solution of problems such as "energy emergency" and"environmental pollution" as well.

Many measures had been taken on trip during peak hours currently, they focused on questions for the moment. It is urgent to establish the theory system of elastic traffic to alleviate and improve urban transportation.

\section{Bi-Level multi-objective programming model based on travel time}

From the application of relative theories, the final optimization results of system can be reflected in the following three aspects.

(1)Minimum of total service time

Targeting at optimization of density, maximization of volume and idealization of speed under elastic equilibrium, model will reaches the anticipated goal of the minimum of total service time.

(2) Maximum of environmental benefits

(3) Minimum of users' travel time

This study divides influence factors into three categories--A (subjective measures), B (objective macrography ) and $\mathrm{C}$ (others, such as economy ,planning). Class $\mathrm{C}$ was not discussed for its uncertainty. 
The model can satisfy user and system optimal, formulated as follows ${ }^{[1]}$ :

(U1) $\min T\left(x_{a}, y_{a}\right)=\sum \sum r_{i} d_{r s} B_{a}{ }^{i}\left(x_{a}(y), y_{a}\right)$

(U2) $\min E=\alpha \sum F\left(x_{a}(y), y_{a}\right)+\beta \sum P\left(x_{a}(y), y_{a}\right)$

s.t. $y_{a} \geqq 0$

Parameters above can be solved by the functions belows:

(L1) $\min Z(x)=\sum \sum \int_{0}^{x_{a}(y)} B_{a}{ }^{i}\left(x_{a}(y), y_{a}\right) d x^{i}$

s.t. $\sum \sum \sum \delta_{r s}^{a k} f_{r s}^{i k}=x_{a}(y), a \in A$

$f_{r s}^{i k} \geqq 0, i=1,2, \ldots, I, k \in P_{r s}, r s \in R S$

$\sum f_{r s}{ }^{i k}=r_{i} d_{r s}, r s \in R S, i=1,2, \ldots, I$

$\sum r_{i}=1$

where, $N-$ set of all nodes.

$A-$ set of all sections.

$R-$ set of all origins.

$S-$ set of all destinations.

$P \_$set of all paths.

$a-$ one section.

$r$ - one origin.

$s \_$one destination.

$k \_$one path.

$i$ - classification of travelers: $i=1$ bus, $i=2$ compact car, $i=3$ non-motor.

$r_{i}-$ proportion of trip mode.

$x_{a}-$ volume of section $a$.

$q-$ OD demand on section.

$d_{r s} \quad$ traffic demand of OD (r-s ).

$f_{r s}{ }^{i k} \_$volume on path $k$ of trip mode $i$ for OD (r-s ).

$x_{a}(y) \_$corresponding volume when designed capacity increment changes.

$y_{i}-$ vector of the of designed capacity increment for section $i$, the decision variable in the bi-level programming model.

$B_{a}{ }^{i}\left(x_{a}(y), y_{a}\right) —$ travel-time of traveler $i$ on section $a$.

$P_{r s} \longrightarrow$ set of sections for all OD.

$F\left(x_{a}\right)$ - fuel consumption for volume of section $a$.

$P\left(x_{a}\right)$ emission for volume of section $a$.

$E$ environmental optimal value.

$\alpha, \beta$ - coefficient.

$\delta_{r s}{ }^{i k}$ relation between path and section, if section $a$ on path $k \delta_{r s}{ }^{i k}=1$, otherwise $\delta_{r s}{ }^{i k}=0$.

$x-$ column vector $\left(\ldots, x_{a}, \ldots\right)^{\mathrm{T}}, a \in A$.

Upper question has two objective functions, $\mathrm{U} 1$ is the network design of elastic equilibrium, which analysis of elastic demand and measures were conducted. U2 is the design of environmental optimizing.

Lower question L1 is users' equilibrium assignment. The travelers' behavior conform to user optimum principle based on the travel time budget.

The steps of Algorithm based on sensitivity is as follows ${ }^{[2]}$ :

Step 1: Set initial solution $y^{0}$, the capacity increment, and set iterations $n=0$.

Step 2: Solve lower question according to given $y^{n}$, obtain the OD demand $q^{n}$.

OD demand. 
Step 4: Substitute the formula $q_{r s} \approx q_{r s}\left(y^{*}\right)+\sum\left[\partial q_{r s}(y) / \partial y_{a}\right]_{y=y^{*}}\left(y_{a}-y_{a}{ }^{*}\right)$ to the upper objective functions, obtain new capacity increment $y^{n+1}$.

Step 5: If $\max \left|y_{a}{ }^{n+1}-y_{a}{ }^{n}\right| \leq \varepsilon$ stop, Where $\varepsilon$ is the iterative precision. Or set $n=n+1$, back to step 2 .

Algorithm ends.

\section{Case Analysis}

The elastic equilibrium of bi-level programming model was validated by public transportation system in PingDingshan (CBD).

Combined with the current situation of PingDingshan, trip mode was divided as follow: A (public transportation), B (compact car) and C (non-motor vehicle).

Bus OD demand in 2016 was Predicted by Growth Factor Method according to the survey data of current travel demand of public transportation. The result was shown as table 1 .

Table 1 Bus OD demand in 2016

\begin{tabular}{cccccc}
\hline D O & $\begin{array}{c}\text { Beidu } \\
\text { Town }\end{array}$ & $\begin{array}{c}\text { Railway } \\
\text { station }\end{array}$ & $\begin{array}{c}\text { ZhanHe } \\
\text { District }\end{array}$ & $\begin{array}{c}\text { Central } \\
\text { Plaza }\end{array}$ & $\begin{array}{c}\text { Dawu } \\
\text { Intersection }\end{array}$ \\
\hline Beidu Town & 3114 & 7898 & 5103 & 10926 & 1785 \\
\hline Railway Station & 7512 & 8640 & 9152 & 19885 & 5192 \\
\hline ZhanHe District & 5435 & 10178 & 4015 & 15604 & 4143 \\
\hline Central Plaza & 10752 & 20359 & 14589 & 23431 & 10380 \\
\hline $\begin{array}{c}\text { Dawu } \\
\text { Intersection }\end{array}$ & 2205 & 6499 & 4837 & 12676 & 3553 \\
\hline
\end{tabular}

This study discussed scene one (only B takes effect) and scene two ( $\mathrm{A}$ and B).

In view of current traffic of PingDingshan (1, Zhongxing road north section. 2, Jianshe road west section. 3, Zhongxing road south section. 4, Jianshe road east section), the scheme was expressed as follows:

(1) Increase the departure frequency

Compared to 10 minutes before, add up the departure frequency to 3 minutes.

(2) Temporary bus lane

JianShe Road and ZhongXing Road during the morning and evening peak hour.

(3) Linear control

Optimize the signal control system of JianShe Road.

(4) LED screen

Set up LED screen near the edge of the central area.

(5) prohibiting left turn, bus exclusive-use signal and bus left-turn waiting zone.

Now considering the five kinds of measures above as the influencing factors of A only, apply the given conditions to the bi-level programming model, build raw data network as table 2 shown. Where $t_{1}$ signifies original runtime of the bus on sections for the whole network, $t_{2}$ signifies original runtime of the compact car on sections for the whole network, $T$ signifies total runtime for whole system ${ }^{[3,4]}$.

Table 2 Raw data in the tested network

\begin{tabular}{cccc}
\hline section & $t_{1}(\mathrm{~s})$ & $t_{2}(\mathrm{~s})$ & $T(\mathrm{~s})$ \\
\hline 1 & 157.7 & 113.8 & \\
2 & 414.3 & 338.8 & 1980314.39 \\
3 & 173.5 & 123.4 & \\
4 & 308.1 & 270.1 & \\
\hline
\end{tabular}

The optimized in the road network is shown as table 3. Where: $t_{1}$ signifies improved runtime of the bus on sections for the whole network, $t_{2}$ improved signifies original runtime of the compact car on sections for the whole network, $T$ ' signifies improved the total runtime for whole system, $T$ - $T$ signifies decrement of total time. 
Table 3 Results of design optimization in the tested network

\begin{tabular}{ccccc}
\hline section & $t_{1}{ }^{\prime}(\mathrm{s})$ & $t_{2}{ }^{\prime}(\mathrm{s})$ & $T^{\prime}(\mathrm{s})$ & $T-T^{\prime}(\mathrm{s})$ \\
\hline 1 & 129.8 & 77.7 & & \\
2 & 174.5 & 150.0 & & \\
3 & 163.9 & 81.8 & 1008346.30 & 971968.09 \\
4 & 187.6 & 226.6 & & \\
\hline
\end{tabular}

Be seen from the chart, travel time of compact car user decreases $0 \sim 3$ minutes averagely, 0 2 minutes to bus.

Then consider the second objective environmental optimum in upper model. The result was shown as table 4 via calculation, where $\mathrm{F}$ signifies the decrement of energy, units by $\mathrm{L} /(\mathrm{km} \times \mathrm{h})$. P signifies the decrement of pollution, namely the emissions of every ton standard coal, units by $\mathrm{t} /(\mathrm{km} \times \mathrm{h})^{[5]}$.

Table 4 Results of environmental optimizing

\begin{tabular}{cccccc}
\hline \multirow{2}{*}{ section } & Decrement & Decrement of & \multicolumn{2}{c}{ variation of volume } & \multirow{2}{*}{ E } \\
\cline { 4 - 5 } & of F L/km & P t/km & Compact car & bus & \\
\hline 1 & 2.26 & 0.73 & -185 & +14 & 1.88 \\
2 & 21.14 & 1.41 & -349 & +20 & 15.21 \\
3 & 5.68 & 0.68 & -168 & +9 & 4.43 \\
4 & 25.36 & 1.41 & -336 & +11 & 19.37 \\
\hline
\end{tabular}

Through the contrast and statistical analysis, it can be found that after those Elastic Equilibrium measures, the traffic congestion gets relief, the amount of compact car effectively decreases, the amount of large-capacity traffic tools increases "Bus Priority" promotes, the energy consumption and emissions of pollutant caused by traffic congestion both decrease, system achieves equilibrium.

\section{Results and Discussion}

The summary of innovations in this dissertation is as following:

1) Application of $\mathrm{ABC}$ classification, trip mode and the influence factors were split by $\mathrm{ABC}$ classification, to divide of elastic equilibrium and discuss respectively according to the specific situations.

2) The optimization of time and environment. The minimum of system time and environmental optimization as the upper objective functions of bi-level programming model, and minimum of users time as the lower.

3)Application of "Elastic Equilibrium". "Elastic Equilibrium" is defined as the new balanced subjected to some elastic factors. The study builds elastic equilibrium system of the urban traffic oriented to "Bus Priority" and showed the expected effect through modeling and simulation.

4)VISSIM simulation. The software VISSIM was adopted to realize the simulation of the volume before and after the implementation of elastic plan in urban areas of PingDingshan. 


\section{References:}

[1] Yang Hai. Sensitivity Analysis for the Elastic-Demand Network Equilibrium Problem with Applications[R]. Transportation Research, 1997, 31 (1):55-70

[2] Ben-Ayed O, Blair Ce. Computational difficulties of Bilevel linear Programming[J]. Operations Research,1990,38 (1):556-559

[3] Ren Gang. Traffic Distribution Model and Algorithm under the Control of Management[M]. Southeast University Press, 2007

[4] Teng Chunsheng, Li Zhihui. Theory and Application of Bi-Level Planning[M]. Science Press, 2002

[5] Pei Yulong, Zhang Yaping. Modeling and Simulation of Traffic System[M]. China Communications Press, 2004 